\title{
Advice on driving while under the care of a crisis resolution team: findings from two audits
}

\author{
Joanna Curwen, ${ }^{1}$ Amy Jebreel ${ }^{1}$
}

The Psychiatrist (2012), 36, 424-426, doi: 10.1192/pb.bp.111.035923

${ }^{1}$ Camden and Islington NHS Foundation Trust, London

Correspondence to Joanna Curwen (joanna.curwen@candi.nhs.uk)

First received 11 Jul 2011, final revision 30 Apr 2012, accepted 14 May 2012

\begin{abstract}
Aims and method To examine the documentation regarding patients driving while under the care of a crisis resolution team and whether advice is being given by all members of the team. An audit was carried out looking at the case records of patients and information was collated about whether they had been asked if they were driving and whether any advice had been given. Following the audit there was a team presentation and guidelines distributed. The audit was repeated 6 months later.
\end{abstract}

Results The first cycle of the audit included 58 patients. There was documentation about driving for two patients and appropriate advice given for one. The second cycle included 53 patients. There was documentation about two patients and advice given for one.

Clinical implications Patients are not being asked whether they drive and advice is not routinely being given despite clear guidelines. Further research is needed to look at interventions at a local level that could improve this.

Declaration of interest None.
Mental illness, alcohol and drugs can impair driving, which can cause accidents. License holders have a legal responsibility to inform the Driver and Vehicle Licensing Agency (DVLA) if they have an illness or disability that might affect their ability to drive (Road Traffic Act 1988). At a Glance Guide to the Current Medical Standards of Fitness to Drive provides guidance to doctors and health professionals. ${ }^{1}$ The guidelines are intended to assist doctors in advising their patients whether or not they should inform the DVLA of their medical condition and what the likely outcome might be. When patients have not informed the DVLA and continue to drive despite being advised of the risks, doctors have a duty to break confidentiality and contact the DVLA themselves. The General Medical Council (GMC) advises doctors to seek guidance from the DVLA if they are unsure about a patient's fitness to drive. ${ }^{2}$ The GMC advises that although the driver is legally responsible for informing the DVLA if they have a condition that may impair their driving, the doctor must explain this to their patient. If the patient refuses to accept the diagnosis or the effect of the condition on their driving, they can be helped to find a second opinion but advised to stop driving in the meantime. If they continue to drive when they might not be fit to do so, every effort must be made to persuade them to stop. If they continue to drive, the doctor should contact the DVLA and disclose the medical condition. The doctor should inform the patient before doing so and also put it in writing to the patient. $^{2}$ The Royal College of Psychiatrists reiterates this guidance. $^{3}$

There have been several studies looking at psychiatrists' knowledge of the DVLA guidelines and their attitudes towards giving advice using questionnaires. They have all demonstrated a lack of knowledge of existing guidelines and a reluctance to advise patients to inform the DVLA where appropriate. $^{4-6}$ Past research has also focused on advice given to psychiatric in-patients by doctors. Orr \& Elworthy looked at documentation of driving advice in the medical case notes of patients admitted for an acute psychotic episode. ${ }^{7}$ They found a slight improvement in documentation after DVLA guidelines were circulated to medical staff but this was only from 0 to $11 \%$.

Management of acute mental illness has changed over the past 10 years. Crisis resolution and home treatment teams may offer an alternative to in-patient admission, with patients increasingly being treated in the community by multidisciplinary teams. Patients under a crisis team may not see a doctor during their treatment episode and therefore other mental health professionals are responsible for ensuring that risks are identified and managed. Nurses also have a responsibility to disclose information that a patient is driving if the individual has been advised to contact the DVLA and not done so. ${ }^{8}$ An audit was carried out looking at the multidisciplinary case records of patients treated by an inner-London crisis resolution team. Information was collated about whether they had been asked if they were driving and whether any advice had been given.

\section{Method}

In the first audit cycle, multidisciplinary case records of all the patients taken on by an inner-London crisis resolution team from 1 May 2009 until 30 June 2009 were examined. 
Patients were excluded if they were admitted immediately after the initial assessment by the crisis resolution team and if they had only been seen once. Patients admitted immediately after initial assessment were excluded as they were no longer under the crisis resolution team and patients only seen once were excluded as they were not considered to be appropriate for the crisis resolution team and therefore not taken on.

All entries on the electronic clinical record system 'RiO' were examined (this is an electronic system that contains the multidisciplinary records for all patients in the team). When patients were taken on by the team following discharge from hospital the records for the recent admission were also reviewed as they could contain information about their driving that could be used by the crisis resolution team. Case records were reviewed looking for any evidence that they had been asked whether they were currently driving and, if so, whether any advice had been given.

All patients are required to have a risk assessment form on RiO completed in their case records. This form includes the heading 'Driving and Road Safety' with a 'Yes or No' tick box underneath for 'last 6 months' and 'ever' and space underneath for any comments.. This box was therefore examined for any documentation that the patient was driving or any advice that had been given. Case records were reviewed looking for the diagnosis of each patient documented at the time of the audit.

Following the audit the findings were presented to the team with a teaching session on the DVLA guidelines and responsibilities of health professionals. The guidelines were also circulated to all team members. The audit cycle was immediately repeated from 5 January 2010 to 28 February 2010. Case records were reviewed in the same way as the first cycle.

\section{Results}

In audit cycle 1, 58 patients were included. Two had specific documentation about driving. One patient who was suicidal had mentioned she was studying to be a taxi driver but there was no documentation of any advice given. The other patient had a diagnosis of mania and was given appropriate advice not to drive. However, 38 patients had the 'Driving and Road Safety' box ticked on the risk assessment but there was no additional information about driving.
In audit cycle 2, 53 patients were included. Two had clear documentation relating to their driving status. Appropriate advice was given about driving to one patient who had been admitted with a manic episode. This advice was given by the ward consultant just prior to discharge rather than the crisis team. This consultant was not part of the crisis team. For the second patient it was documented that the patient was not driving as his car had broken down. For 33 patients the risk assessment box for driving/road safety was completed but again there was no information about driving.

Psychiatric diagnoses of the patients in each audit are shown in Table 1. The diagnoses have been grouped into ICD-10 categories. ${ }^{9}$ Schizophrenia and other psychotic disorders and mood disorders were the most common diagnoses, $69 \%$ in audit cycle 1 and $60 \%$ in audit cycle 2 . Very few patients were deemed to have no evidence of mental illness: $2 \%$ in audit cycle 1 (if no diagnosis documented is assumed to be no evidence of mental illness) and $8 \%$ in audit cycle 2 .

\section{Discussion}

There was no improvement in the recording of information about driving or advice given in the case notes in the second audit cycle compared with the first audit cycle. In the first audit the appropriate advice was given to one patient by a nurse in the crisis team and in the second audit the advice was given by the ward consultant prior to discharge and not a member of the crisis team. The section on the risk assessment form for driving/road safety on RiO did not add useful information about whether the patient was driving. Ticking 'Yes' on the form could be for several reasons. It could mean that the patient is driving or that the patient has directly caused an accident while driving. However, it could also mean that the patient has been a passenger in a car involved in an accident or been hit by a car while crossing the road. Unfortunately, the majority of patients had no additional comments to explain why the box was ticked 'Yes'.

The At a Glance Guide to the Current Medical Standards of Fitness to Drive ${ }^{1}$ contains guidelines for depression, anxiety, mania, hypomania, acute and chronic psychotic disorders and personality disorders. The majority of patients in the audits had one of these conditions. Many patients with these conditions are prescribed sedating

\begin{tabular}{|c|c|c|}
\hline & \multicolumn{2}{|c|}{$n(\%)$} \\
\hline & Audit $1(n=58)$ & Audit $2(n=53)$ \\
\hline Psychoactive substances (F10-19) & $4(7)$ & $8(15)$ \\
\hline Schizophrenia, schizotypal, delusional (F20-29) & $18(31)$ & $15(28)$ \\
\hline Affective (F30-39) & $22(38)$ & $17(32)$ \\
\hline Neurotic, stress-related and somatoform (F40-48) & $5(9)$ & $3(6)$ \\
\hline Specific personality disorders (F61) & $8(14)$ & $6(11)$ \\
\hline No evidence of mental disorder/illness & $1(2)$ & $2(4)$ \\
\hline No documentation of presence or absence of mental disorder/illness & $0(0)$ & $2(4)$ \\
\hline
\end{tabular}


medication that can also impair driving. A limitation of the audit is that the diagnoses were made by different psychiatrists of differing grades and not using standardised instruments. Despite this, it is very worrying that mentally ill patients under the care of the crisis team may pose a risk to themselves or others if they are driving and this is not identified by the mental health professionals caring for them. These findings suggest that the presentation to the team and dissemination of driving guidelines had little impact on the practice of team members. The way in which a team responds to new processes or structures being introduced is a very important factor and resistance to change is not uncommon. If documentation on driving was a key performance indicator, managers would be accountable for ensuring this information was obtained by their staff.

It is possible that the lack of documentation regarding advice may not equate with a lack of advice given, however, it is the documentation that is of importance when records are being reviewed by the legal profession, judges and coroners. It is possible that health professionals may avoid discussing driving with patients, since once a patient reveals he is driving there is a responsibility to ensure that he informs the DVLA. Culshaw et al suggest that health professionals may be avoiding such discussions in the fear that it may adversely affect the doctor-patient relationship. ${ }^{10}$

This reluctance to ask patients about driving and give appropriate advice was found across 1519 health professionals across all specialties in a report published by the Department for Transport. ${ }^{11}$ There may also be a wider uncertainty about which health professionals have responsibility to give advice to patients about driving. In this report by the Department of Transport, 101 health professionals interviewed were asked whose responsibility it is to advise patients on driving; most non-doctors thought that doctors had the responsibility. Most doctors thought that the whole team should be responsible and that the first person to come into contact with an unfit driver should offer advice. The Code gives clear guidelines to nurses that they must disclose information if there is a risk of harm, which indicates that nurses have a duty to inform the DVLA if a patient is driving and has not informed them themselves. ${ }^{8}$ The guidelines for allied health professionals are less clear but they will still have a duty of care towards their patient that should extend to an awareness of the issues around driving.

\section{Conclusions}

All healthcare professionals should be aware whether their patients are driving and be giving them the appropriate advice if they are. Crisis resolution teams may be caring for individuals who are hypomanic, acutely psychotic, suicidal or taking medications that may impair their ability to drive and therefore should not be driving. Although more training may be required to improve awareness, in our study this was insufficient to make a difference to the results found in the second audit.

Changing the risk assessment form on RiO so that there is a specific question asking whether the patient is driving would greatly increase the likelihood of this being addressed. Unfortunately, this cannot be implemented locally and could take a long time to change. Therefore the focus for making a difference must be increasing awareness among staff and patients of the issues around driving and mental illness. A poster regarding the guidelines for driving with psychiatric illness could be placed in outpatient clinics, on wards and also where health professionals are working, which could increase awareness among both patients and staff. Further useful research could explore whether this is an effective intervention.

\section{Acknowledgements}

Thank you to Dr Koye Odutoye, Dr John Dunn and Dr Bhaskar Punukollu for their advice and support.

\section{About the authors}

Joanna Curwen and Amy Jebreel are specialist trainees in general adult psychiatry at Camden and Islington NHS Foundation Trust, London.

\section{References}

1 Driver and Vehicle Licensing Agency. At a Glance Guide to the Current Medical Standards of Fitness to Drive. TSO (The Stationery Office), 2011.

2 General Medical Council. Confidentiality: Reporting Concerns about Patients to the DVLA or DVA. GMC, 2009.

3 Royal College of Psychiatrists. Good Psychiatric Practice: Confidentiality and Information Sharing (2nd edn) (College Report CR160). Royal College of Psychiatrists, 2010.

4 Wise MEJ, Watson JP. Postal survey of psychiatrists' knowledge and attitudes towards driving and mental illness. Psychiatr Bull 2001; 25: 345-9.

5 Thompson P, Nelson D. DVLA regulations concerning driving and psychiatric disorders. Knowledge and attitudes of psychiatrists. Psychiatr Bull 1996; 20: 323-5.

6 Humphreys SA, Roy L. Driving and psychiatric illness. Psychiatr Bull 1995; 19: 747-9.

7 Orr EM, Elworthy TSE. Audit of advice on driving following hospitalisation for an acute psychotic episode. Psychiatr Bull 2008; 32 $106-7$.

8 Nursing \& Midwifery Council. The Code. NMC, 2008.

9 World Health Organization. The ICD-10 Classification of Mental and Behavioural Disorders: Clinical Descriptions and Diagnostic Guidelines. WHO, 1992.

10 Culshaw M, Wootton L, Wylie S. Alcohol dependence and driving: a survey of patients' knowledge of DVLA regulations and possible clinical implications. Psychiatr Bull 2005; 29: 90-3.

11 Hawley C. The Attitudes of Health Professionals to Giving Advice on Fitness to Drive. Department for Transport, 2010. 